\title{
OBSERVATION OF CHANGING OF THE INTERNAL CONVERSION COEFFICIENT UNDER MÖSSBAUER EFFECT AT MAGNETIC TRANSITION IN Rh-Fe SYSTEM
}

\author{
T. Ruskov \\ Institute of Nuclear Research and Nuclear Energy, Tsarigradsko shosse 72, 1784, Sofia, Bulgaria
}

(Received March 13, 1998)

\begin{abstract}
The magnetic disorder-order transition in the Rh-Fe alloy is studied by conversion electron Mössbauer spectroscopy. The drastic increase of the area under the Mössbauer spectrum at the transition from the paramagnetic to the magnetic state could be explained by diminishing the internal conversion coefficient. Thus our experimental results directly confirm the theory of the collective effect in the system of radiating nuclei developed by Yukalov [11].
\end{abstract}

Key words: Mössbauer effect, magnetic transition, conversion coefficient, superfluorescence.

PACS number(s): 76.80.+y, 23.20.Nx, 75.40.-s, 78.45.+h, 42.50.Fx, 42.50.Gy

\section{INTRODUCTION}

There were many experiments in which a strong increase of the area under the Mössbauer spectrum below the transition temperature from the paramagnetic to the magnetic state was observed [1-3]. After a correction due to the saturation effect the increase of the area under Mössbauer spectrum below the Curie point $T_{c}$ as compared to the spectrum area in paramagnetic state reached $20 \div 50 \%$. They attempted to find the explanation of this phenomenon in the abrupt changes of the phonon characteristics (a strong increase of the Debye temperature and thus of the Debye-Waller factor) when the material passes to the magnetic order state. This explanation however contradicts other experiments such as neutron scattering, sound velocity measurements, etc. where no lattice anomalies have been observed passing from a paramagnetic to a magnetic state.

It should be noticed that the area change under the Mössbauer spectrum was observed only in the so called absorption experiment when the investigated material represented the absorber in the Mössbauer absorption geometry or a scatterer in the scattering geometry. Very interesting results were obtained in the paper of Babikova et al. [4] in which two Mössbauer experiments were described on a $\mathrm{Ni}^{57} \mathrm{Fe}$ sample in the range of the Curie point $\left(T_{c}=625 \mathrm{~K}\right)$. In these experiments the temperature dependence of the Mössbauer spectrum area was measured in two ways: by using $\mathrm{Ni}{ }^{57} \mathrm{Fe}$ sample $\left(1.2\right.$ at $\left.\%^{57} \mathrm{Fe}\right)$ as an absorber (the single line source ${ }^{57} \mathrm{CoCr}$ was at room temperature) and by using a ${ }^{57} \mathrm{CoNi}$ $(1.2$ at $\% \mathrm{Co})$ as a source while the single line stainless steel absorber was at room temperature (source experiment).

It should be noticed that the first experiment showed a sharp increase of the area under the spectrum below Curie point while at the second experiment when a radioactive source was incorporated into the $\mathrm{Ni}$ material no magnetic anomalies were observed. This second experiment casts doubt on the reason that such magnetic anomaly could be found in the drastic change of the phonon characteristics of the samples. Of course there is some difference between the two samples: the Mössbauer absorber $\mathrm{Ni}{ }^{57} \mathrm{Fe}\left(1.2\right.$ at $\left.\%^{57} \mathrm{Fe}\right)$ and the source $\mathrm{Ni}{ }^{57} \mathrm{Co}$ $(1.2$ at \% Co), but it is most unlikely that the phonon characteristics of the sample depends on the impurity element (Fe or Co) so strongly.

That is why the authors of ref. [4] proposed that the drastic change of the Mössbauer spectrum area below the Curie point was not related to the change of the DebyeWaller factor $f^{\prime}$, but to a change of the resonance cross section $\sigma_{0}$ through the change of the conversion coefficient, because the conversion coefficient is the only quantity in the expression of $\sigma_{0}$ which could depend on the behavior of the electrons during a magnetic transition.

Later, Kolk et al. [5] carried out a research on temperature dependence of the recoilless fraction near the Curie point for a sample of ${ }^{57} \mathrm{Co}$ in a matrix of $100 \%$ ${ }^{56} \mathrm{Fe}$ (source experiment). The authors did not observe any abrupt change of the spectrum area (respectively of the Debye-Waller factor of the source $f_{s}$ ) at $T_{c}$ but a small deflection of $d f_{s} / d T$ at this point was observed. It should be noticed however, that two different methods have been used to measure the $f_{s}(T)$ dependence below and above Curie point respectively. On the other hand the experimental errors in the $f_{s}(T)$ dependence were rather high, so the observed deflection at the Curie point is not evident.

Dvoretski et al. [6] did not observe any temperature dependence jump of the ${ }^{57} \mathrm{CoNi}$ Mössbauer emission spectrum area at the passage through the Curie point as well. In addition they had measured the $X$-ray and $\gamma-$ radiation energy spectrum of a ${ }^{57} \mathrm{CoNi}$ for the temperatures above and below the Curie point. The relative peak intensities of the $X$-rays and $\gamma$-radiation did not change more than $1 \%$ at the Curie point. That means the conversion coefficient of the $14.4 \mathrm{keV} \gamma$-radiation of a ${ }^{57} \mathrm{Fe}$ nucleus does not change at the magnetic transition either.

Summarizing the above mentioned experiments, one can say: the area under Mössbauer spectrum changes when one passes trough Curie point only in an absorp- 
tion experiment. As the area depends both on the recoilless fraction and on the resonance cross-section of the absorber it is not possible to determine only from the experiment which quantity is changed $f^{\prime}$ or $\sigma_{0}$. There is not any area change when passing through the Curie point in the source experiment. That means the recoilless fraction does not change because the area in this case depends only on $f_{s}$ ( $\sigma_{0}$ is related to the absorber). On the other hand confusing circumstance is the constancy of the conversion coefficient when a radioactive source passes from a paramagnetic to a magnetic order state.

Standing on neutron scattering experiments, sound velocity measurements, etc., Yukalov [11] has shown that the Debye-Waller factor and the Mössbauer effect probability did not change more than $1 \%$ due to the magnetic transition. So, no magnetic anomalies of the spectrum area are to be expected due to the changing of this quantity.

In what follows we shall describe an absorption experiment in which the abrupt increase of the spectrum area below the Curie point could be explained directly by a change of the resonance cross-section (conversion coefficient) without turning to any theoretical consideration or other sort of experiments.

\section{THE EXPERIMENT}

To avoid thickness saturation effect a conversion backscattering electron Mössbauer spectroscopy was used. In this case the surface and the layers in the immediate vicinity of the surface should work.

For the $14.4 \mathrm{keV}$ resonance line of ${ }^{57} \mathrm{Fe}$, the $K$-shell conversion electrons of $7.3 \mathrm{keV}$ with the subsequent $5.6 \mathrm{keV}$ Auger electrons give the main contribution to the back-scattered resonance spectrum. The contribution of the $L, M$ shells conversion electrons with the energy $13.56 \mathrm{keV}$ and $14.32 \mathrm{keV}$ respectively is only $6 \%$ of the $K$-shell group. Concerning the $L-M M$ Auger electrons, due to their small energy $(0.5-0.6 \mathrm{keV})$, they could be neglected.

The range of the $K$-shell group of electrons in iron is not more than $2000 \AA$, but due to multiple scattering and nearly isotropic angular distribution actually $90 \%$ of them emerge from a surface layer of a thickness not greater than 1000-1200 $\AA$ [12]. Registering conversion electrons with definite energy, one could make depth selective analysis of the surface layer. It is obvious that the higher the energy of conversion electrons the more close to surface they are emitted. Thus we could select a very thin layer of the sample less than $300 \AA$ to avoid any thickness effect.

A Rh-30 at $\%{ }^{57} \mathrm{Fe}$ sample was chosen to study the area change under the electron conversion Mössbauer spectrum when the sample passes from a paramagnetic to an antiferromagnetic state. In fig. 1 one can see the lower part of a helium cryostat together with a proportional counter for the conversion electrons working in back-scattering geometry. A gas chamber made of stainless steel, in the bottom of which are mounted, first the sample and then the proportional counter, is attached to the helium vessel of the cryostat.

The proportional counter has the form of a cylinder $10 \mathrm{~mm}$ high and $56 \mathrm{~mm}$ in diameter. It is a multiwire system consisting of two cathode and one anode grids. The grids are stretched over washers of a foiled fibre glass reinforced plastic. Both the anode grid and the cathode grids are made of a gold-plated tungsten wire, $20 \mu \mathrm{m}$ for the anode and $50 \mu \mathrm{m}$ for the cathode. The number of anode wires is two or three. The distance between the two adjacent cathode wires is $2 \mathrm{~mm}$. The assembled counter is placed before the sample and the distance between the counter and the sample is $3 \mathrm{~mm}$. By this means it is possible to isolate electrically the proportional counter from the body of the gas chamber and from the sample. The gas chamber is filled with the He of $99.98 \%$ purity to $1 \mathrm{~atm}$ pressure at room temperature. If one applies to the cathode grids several tens of volts of negative potential with respect to the body of the gas chamber, the anode-cathode potential difference and hence, the gas gain will increase.

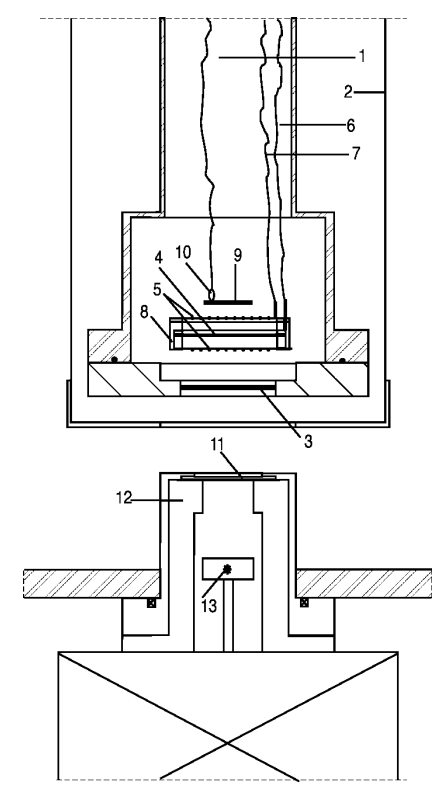

Fig. 1. Proportional counter set up: 1 - gas chamber, 2 - $77 \mathrm{~K}$ copper shield, 3 - aluminized Mylar window, 4 anode grid, 5 - cathode grids, 6 - anode high voltage supply, 7 - cathode voltage supply, 8 - teflon counter body, 9 - sample, 10 - temperature control, 11 - lucite window, 12 - lead shielding and collimator, $13-\gamma$-source.

It was theoretically demonstrated that a rather large part of the emitted resonant and non-resonant electrons had the energy under $50 \mathrm{eV}$ [13]. Obviously these electrons will be stopped by cathode grids at an appropriate negative potential. Of course, the $3 \mathrm{~mm}$ He layer between the sample and the counter will additionally slow down the electrons emitted from the sample. The mean en- 
ergy lost of an electron in this gas layer is about $4 \mathrm{keV}$. Usually, when lowering the temperature the gas amplification of the proportional counter decreases. So, only the conversion electrons which are emitted from a very thin layer at the surface of the sample contribute to the conversion electron Mössbauer spectrum in the case. Our evaluation of the thickness of this layer is less than $300 \AA$.

Variation of the gas amplification leads to a change of the resonance effect and of the area under the resonance curve [14]. That is why a reference Mössbauer scatterer (an iron foil enriched with $90 \%{ }^{57} \mathrm{Fe}$ ) was placed near the $\mathrm{Rh}-\mathrm{Fe}$ sample. In the temperature interval $77 \mathrm{~K}-58 \mathrm{~K}$ the recoilless fraction for a metallic iron practically does not change [15]. There is no magnetic transition for this temperature interval too, so if there is some change of the area under Mössbauer spectrum of the reference scatterer it will be only due to the change of the gas amplification. Using the ratio of the area of the $\mathrm{Rh}-\mathrm{Fe}$ spectrum to the area of an iron spectrum we could eliminate the impact of the gas amplification variation.

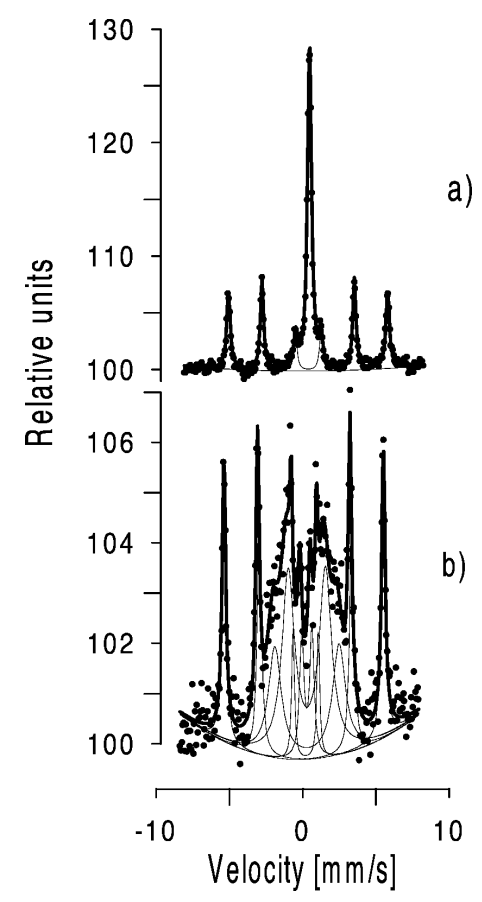

Fig. 2. Conversion electron Mössbauer spectra of Rh-Fe sample + reference Fe scatterer: a) $T=77 \mathrm{~K}$, b) $T=58 \mathrm{~K}$.

In fig. 2 two Mössbauer spectra of the investigated sample and the reference scatterer are shown - at $77 \mathrm{~K}$ and $58 \mathrm{~K}$ respectively. The central line in fig. $2 \mathrm{a}$ corresponds to $\mathrm{Rh}-\mathrm{Fe}$ sample in paramagnetic state. The other six-lines spectrum corresponds to the reference iron scatterer. In fig. $2 \mathrm{~b}$, below the Curie point the $\mathrm{Rh}-\mathrm{Fe}$ spectrum appears as a not completely resolved six-lines spectrum.

\section{EXPERIMENTAL RESULTS AND DISCUSSION}

For a thin scattering layer the area under Mössbauer spectrum could be written as

$$
A=\operatorname{const} \frac{f^{\prime} \alpha}{(1+\alpha)^{2}}
$$

where $f^{\prime}$ is the recoilless fraction of the scatterer sample and $\alpha$ is the conversion coefficient. The obtained ratio of the normalized Rh-Fe Mössbauer spectrum areas (to the reference absorber) below and above the Curie point is

$$
\frac{A_{T=58 \mathrm{~K}}}{A_{T=77 \mathrm{~K}}}=1.84 \pm 0.10
$$

The question is whether this drastic change of the area under Mössbauer spectrum below the Curie point could be assigned to the change of recoilless fraction or resonance cross-section (conversion coefficient) ? The experimental value of $f$ for ${ }^{57} \mathrm{Co}^{-57 *} \mathrm{Fe}$ impurity atoms in $\mathrm{Rh}$ at room temperature is 0.78 and at $4.2 \mathrm{~K}-0.87$ [15]. For $77 \mathrm{~K}$ we obtain $f=0.85$ [15]. For metallic iron (source experiment) the experimental value for $f$ at $77 \mathrm{~K}$ is $0.8 \div 0.9$, so for the alloy $\mathrm{Rh}-30$ at $\%{ }^{57} \mathrm{Fe}$ we could accept $f^{\prime}=0.85$ too. If we assume that the change of the area is produced by the change of the recoilless fraction we should accept that in a magnetic order state $f^{\prime}$ grows up to 1.7 which is impossible as the recoilless fraction is a probability, so it can not be more than unity. Then the next explanation should be sought in a change of the conversion coefficient.

The value of the conversion coefficient in the paramagnetic state is 8.2 [15]. Taking into account the constancy of $f^{\prime}$, from equations (1) and (2) we obtain for the conversion coefficient in a magnetic order state the value 3.2 . Thereby, we have shown directly without assuming any theoretical considerations that in the absorption (scattering) Mössbauer experiment the abrupt change of the spectrum area below the Curie point has to be assigned to the change of the conversion coefficient.

In ref. [11] Yukalov has theoretically considered the possibility of a change of the conversion coefficient when the "resonance atoms" in the absorber pass from a paramagnetic to a magnetic order state. The basic idea is as follows: the system of Mössbauer nuclei in a resonance exciting field (incident and reemitted $\gamma$-quantum) is equivalent to an ensemble of two level emitters. These emitters interact with each other through a virtual photon exchange. The strong hyperfine field in the case of magnetic order aligns the "magnetic dipoles" (emitters), as a result a coherent state is formed, producing a coherent $\gamma$-resonance radiation.

This phenomena of coherent radiation is analogous to the so called superfluorescence first described theoretically by Dicke [7]. Such a phenomena was carefully investigated, both theoretically and experimentally in the op- 
tical range of energies $E_{\gamma} \sim 1 \mathrm{eV} \div 100 \mathrm{eV}$ (see, e.g., the reviews $[8,9]$ ), in which case the wave length $\lambda_{\gamma}$ is much greater than the average distance between the emitters $a \sim 10^{-8} \mathrm{~cm}$.

In the energy range of the $X$ - and $\gamma$-rays the situation is opposite: $\lambda_{\gamma} \leq a$. Nevertheless, in the presence of a strong magnetic field (in the case of $M 1$ transitions) it was shown that the coherent state of emitters can be formed as well [10].

When such a coherent radiation takes place in the system of nuclei, the conversion coefficient of the single nucleus $\left(\alpha_{0}\right)$ transforms into a conversion coefficient of an ensemble of nuclei $\left(\alpha_{c}\right)$ by the equation

$$
\alpha_{c}=\frac{\alpha_{0}}{1+C}
$$

where $C$ is the ratio of coherent to incoherent $\gamma$-radiation part

$$
C=\frac{I_{c o h}}{I_{\text {incoh }}}
$$

In our experiment the corresponding value of ratio (4) is $C \simeq 1.56$ assuming that all the effect of changing of the spectrum area is due to the mechanism discussed. Such value is in accordance with the theoretical estimations $[10,11]$.
The coherent state formation in the system of resonance nuclei is a collective effect which appears in a Mössbauer absorption or scattering experiments. In the case of a Mössbauer source experiment such a collective phenomena is impossible. Indeed, the number of emitters during the lifetime of the $14.4 \mathrm{keV}{ }^{57} \mathrm{Fe}$ exited state which is of the order of $10^{-7} \mathrm{~s}$ is too small to realize the coherent state, so the influence of hyperfine magnetic field can cause only a negligible change of the conversion coefficient of the order of $10^{-5}$ to $10^{-3}$ [11].

To conclude the discussion, we would like to stress that the investigation of the collective effects in the $X$ - and $\gamma$-energy range is an open problem, both theoretically and experimentally. In this respect a very promising tool is the Mössbauer spectroscopy as was demonstrated in this paper. By an appropriate choice of the Mössbauer isotope, of the corresponding matrix, and other parameters one can hope to increase essentially the coherence coefficient $C$ (4) and this circumstance can be used in the construction of a gamma-lasers.

\section{ACKNOWLEDGMENTS}

The author is thankful to Abdallah Rastanawi for his help in the measurements and to Rusko Ruskov for reading the manuscript and fruitful discussions. The work was supported financially by the Bulgarian National Fund "Scientific Investigations".
[1] L. A. Alekseev, P. L. Gruzin, M. N. Uspensky, M. R. Gryaznov, Pis'ma Zh. Eksp. Teor. Fiz. 14, 292 (1971).

[2] V. I. Nikolaev, V. S. Rusakov, Zh. Eksp. Teor. Fiz. 70, 1949 (1976)

[3] V. F. Kumeyshin, O. A. Ivanov, Fiz. Met. Mettaloved. 40, 1295 (1975).

[4] U. F. Babikova, P. L. Gruzin, A. N. Spirin, M. N. Uspensky, Solid State Commun. 32, 191 (1979).

[5] B. Kolk, A. Bleloch, D. Hall, Hyperfine Interactions 29, 1377, (1986).

[6] V.I. Dvoretsky, L. V. Inzhechik, V. G. Tsinoev, Hyperfine Interactions 61, 1415, (1990)

[7] R. H. Dicke, Phys. Rev. 93, 99 (1954).

[8] A. V. Andreev, V. I. Emelyanov, I. A. Ilynski, Usp. Fiz. Nauk 131, 653 (1980).
[9] M. Gross, S. Haroche, Phys. Rep. 93, 301 (1982).

[10] V. I. Yukalov, preprint JINR P17-87-841, Dubna, (1987); preprints JINR P17-88-13, JINR P17-88-14, Dubna, (1988).

[11] V. I. Yukalov, Mod. Phys. Lett. B 3, 1337 (1989).

[12] G. N. Belozerski, Mossbauerovskaia spiektroskopiia kak mietod issliedovaniia povierkhnosti (Mössbauer Spectroscopy as a Method for Surface Study) (Energoatomizdat, Moscow, 1990).

[13] T-S Lee, J. S. Zabinski, B.J. Tatarchuk, Nukl. Instrum. Methods B 44, 107 (1989).

[14] T. Ruskov, G. Passage, A. Rastanawi, R. Radev, Nukl. Instrum. Methods B 94, 565, (1994).

[15] Handbook of spectroscopy, Volume III, edited by J. W. Robinson (CRC Press, 1981). 